\title{
Reflexões Teórico-Metodológicas acerca da Pesquisa em Compreensão de Textos com Crianças
}

\author{
Alina Galvão Spinillo ${ }^{1}$ \\ Luciana Vasconcelos dos Santos Dantas Hodges \\ Universidade Federal de Pernambuco \\ Alberto Santos Arruda \\ Universidade Federal da Paraíba
}

\begin{abstract}
RESUMO - Dada sua natureza multifacetada, a compreensão de textos tem sido investigada a partir de uma grande variedade de recursos metodológicos. O presente artigo tece uma reflexão de natureza teórico-metodológica acerca dos principais métodos de investigação adotados nas pesquisas realizadas com crianças sobre esse tema. A análise recai sobre as principais características desses métodos, seus limites e possibilidades que apresentam ao examinar diferentes aspectos envolvidos na compreensão de textos. Dois parâmetros foram considerados: as unidades linguísticas investigadas e o momento em que a compreensão é avaliada. As análises e discussões conduzidas ilustram a estreita relação entre método e dado, contribuindo para que o pesquisador tenha uma visão mais clara daquilo que os métodos efetivamente permitem examinar sobre a compreensão de textos.
\end{abstract}

Palavras-chave: compreensão da leitura, métodos de pesquisa, crianças

\section{Theoretical-Methodological Discussion on Research in Reading Comprehension with Children}

\begin{abstract}
Due to its multifaceted nature, reading comprehension has been investigated from a variety of methods. This paper focuses on a theoretical-methodological discussion about the methods usually adopted in studies with children. The analysis focused on the main characteristics of these methods, their limits, and possibilities they present in examining different aspects involved in reading comprehension. Two parameters were considered: the linguistic units investigated, and the moment at which comprehension is evaluated. The analyses and discussions conducted illustrate the close relationship between method and data, helping the researcher to have a clearer view of what methods effectively allow the examination about text comprehension.
\end{abstract}

Keywords: reading comprehension, research methods, children

O pensamento científico busca estabelecer relações entre elaborações teóricas e o fenômeno que se deseja investigar. Essa busca evidencia outra relação: aquela entre o método de investigação e o que comumente é chamado dado. A respeito da compreensão de textos em particular, Keenan, Potts, Golding e Jennings (1990) comentam que as opções metodológicas são tão importantes quanto o próprio dado. Brandão e Spinillo (1998) complementam que a escolha metodológica reflete tanto a concepção de compreensão assumida pelo pesquisador como implica na avaliação de um determinado aspecto e não de outros. Isso ocorre porque $\mathrm{o}$ ato de compreender textos é constituído por dimensões indissociáveis (Spinillo, 2013), sendo dificilmente investigado a partir de um único método. Sem pretender realizar um levantamento exaustivo das investigações na área, o foco deste artigo recai sobre uma reflexão teóricometodológica para ilustrar a estreita relação entre método e dado, com vistas a contribuir para que o pesquisador tenha

1 Endereço para correspondência: Universidade Federal de Pernambuco, Pós-Graduação em Psicologia Cognitiva, Centro de Filosofia e Ciências Humanas, $\mathrm{CFCH}, 8^{\circ}$ andar, Cidade Universitária, Av. da Arquitetura, s/n, Recife, PE, Brasil. CEP: 50.740-550.E-mail: alinaspinillo@ hotmail.com uma visão mais clara daquilo que os métodos ${ }^{1}$ permitem efetivamente examinar a respeito da compreensão de textos. Para isso, optou-se por agrupar os métodos em função de dois parâmetros. O primeiro, mais usual, toma por base as unidades linguísticas que são alvo de análise nas pesquisas: a palavra, a sentença e o texto como um todo. O segundo, mais recente e inovador, considera o momento em que a compreensão é avaliada: durante ou após a leitura do texto.

\section{Os Métodos de Pesquisa em Função das Unidades Linguísticas Investigadas}

A opção de agrupar os métodos em função da unidade linguística que focalizam se justifica porque os significados atribuídos ao texto são gerados a partir de uma rede de relações lexicais, semânticas, sintáticas, pragmáticas e estruturais nele configuradas. Essa materialidade linguística do texto associada ao conhecimento de mundo do leitor permite a articulação entre níveis de conhecimento que são processados a partir de vias de processamento ascendente

1 Os métodos aqui discutidos são adotados em pesquisas, não sendo considerados testes estandardizados que avaliam a compreensão em grande escala cujos resultados são comparados com uma amostra normativa. 
e descendente (Cain \& Oakhill, 2004; Colomer \& Camps, 2002; Kleiman, 2002). Ressalta-se que investigar uma dada unidade linguística não significa que o processamento em relação às demais unidades tenha sido suspenso. Na realidade, o que o recurso metodológico permite é focalizar uma dessas unidades.

Quando a unidade linguística é a palavra, alguns métodos avaliam o acesso lexical, como o método de reconhecimento, identificação ou nomeação de palavras. O procedimento envolve a leitura de palavras em um texto (Forster, 1981; Keenan et al., 1990; Oakhill \& Cain, 2007) ou de palavras isoladas (Salles \& Parente, 2002). O tempo de leitura é considerado uma medida importante, pois o tempo de nomeação de uma palavra cujo significado esteja associado ao texto lido é maior do que o tempo necessário para nomear uma palavra que não esteja semanticamente relacionada ao texto ou uma palavra desconhecida. Muitos dos estudos investigam o vocabulário (Stanovich, West, Cunningham, Cipielewski \& Siddiqui, 1996) ou a capacidade de decodificação (Balota, 1990; Gough, Hoover, \& Peterson, 1996; Oakhill \& Cain, 2007; Yuill \& Oakhill, 1991). Esses aspectos são importantes, visto que limitações no vocabulário ou problemas na decodificação geram dificuldades em reconhecer palavras e em integrar informações que permitam atribuir significados apropriados ao texto.

Outro método amplamente utilizado é o teste ou técnica de cloze (Cain \& Oakhill, 2004; Drum, Calfee, \& Cook, 1981; Santos, Primi, Taxa, \& Vendramini, 2002; Tunmer, Nesdale, \& Wright, 1987). Santos, Boruchovitch e Oliveira (2009) ressaltam ser esse tanto um instrumento de diagnóstico como de intervenção na superação de dificuldades de compreensão. A técnica consiste em preencher com palavras as lacunas em um texto escrito, requerendo do leitor integrar a informação veiculada antes com a informação veiculada após a lacuna, para que a palavra inserida garanta a continuidade de sentidos. As pistas para o preenchimento das lacunas são de natureza semântica (garantir o sentido do texto) e sintática (respeitar regras da língua).

Há inúmeras variações dessa técnica, como documentam Oliveira, Boruchovotch e Santos (2009): cloze lexical, cloze gramatical, cloze labirinto, cloze cumulativo, cloze pareado, cloze restringido, cloze com chaves de apoio, cloze pósleitura oral e cloze interativo. O teste cloze cumulativo toma por base uma única palavra, que deve ser inserida em todas as lacunas. Essa repetição pode levar o leitor a não mais ler o texto e apenas inserir automaticamente a palavra apropriada. Dentre as versões de múltipla escolha, há variações relativas à forma de apresentar as alternativas. Uma limitação dessas variações é que a alternativa correta pode ser escolhida apenas com base no preenchimento de algumas poucas lacunas. Além disso, as escolhas podem ser feitas com base em exclusões de possibilidades e não a partir de inferências.

Há versões cuja aplicação é individual, como o cloze pós-leitura oral e o cloze interativo. No cloze pós-leitura oral, solicita-se que todo o texto seja lido em voz alta pelo indivíduo antes que inicie o preenchimento das lacunas. $\mathrm{Na}$ versão interativa, solicita-se justificar a escolha de preenchimento de cada lacuna. Essas versões geram dados bastante informativos acerca da compreensão e superam limitações das versões anteriores.
A técnica de cloze lexical e gramatical com escolha livre é a que mais envolve o estabelecimento de inferências e o uso de informações intra e extratextuais. $O$ formato livre permite a atribuição de significados que não foram definidos pelo pesquisador a priori, havendo margem para que o leitor traga seu conhecimento de mundo para a situação de leitura. De modo geral, a técnica de cloze demanda habilidades sintática e semântica, bem como pouco esforço da memória, visto que o texto está disponível no momento do preenchimento das lacunas. Segundo Oliveira, Boruchovitch e Santos (2007), o preenchimento de cada lacuna pode ser analisado a partir de uma correção literal ou de uma correção por sinonímia. A correção literal aceita apenas como correta a palavra definida como aceitável e desde que grafada corretamente. A correção por sinonímia aceita como correta uma palavra que tenha o mesmo significado que a palavra considerada correta pelo pesquisador. A correção literal nos parece problemática, pois limitações ortográficas poderão confundidas com dificuldades de compreensão. O segundo tipo de correção, de natureza essencialmente semântica, aproxima-se da concepção de que o leitor tem papel ativo na compreensão e de que o texto pode ter vários significados. A correção literal parece se distanciar destas concepções que permeiam os modelos de compreensão de textos mais adotados na literatura, como o de Kintsch (1998). A técnica de cloze não pode ser aplicada a pessoas que não dominam a leitura e a escrita, como crianças em anos escolares iniciais ou adultos pouco escolarizados.

Dadas suas características, nota-se que a técnica de cloze é um híbrido, pois embora verse sobre a palavra, requer do leitor integrar passagens do texto localizadas imediatamente antes e após a lacuna a ser preenchida. Outro ponto a ser comentado é que esta técnica envolve uma leitura interrompida do texto, situação esta denominada de metodologia on-line, como discutido adiante. Porém, diferentemente de outras metodologias on-line, essa técnica oferece pouca margem para explorar o estabelecimento de inferências de maneira mais ampla, pois a cloze se volta mais para o estabelecimento de inferências locais.

Quando a unidade linguística é a sentença, o método de reconhecimento é bem aceito entre os pesquisadores, consistindo em determinar se uma sentença estaria ou não presente em um texto ou se determinada sentença seria verdadeira ou falsa em relação ao texto (Yuill \& Oakhill, 1991). Há estudos que comparam o tempo de leitura de sentenças-alvo presentes em um texto de forma implícita com o tempo de leitura de sentenças-alvo presentes de forma explícita (Haviland \& Clark, 1974; Keenan et al., 1990). Observa-se que o tempo de leitura é maior quando a sentença lida (ou ouvida) corresponde a uma inferência (informação implícita) do que quando corresponde ao que estava explícito no texto (informação literal), pois estabelecer inferências demanda mais tempo do leitor.

Uma maior participação dos aspectos extratextuais é observada, contudo, quando recursos metodológicos incidem sobre o texto. Isso pode ser verificado em pesquisas em que o indivíduo é solicitado a fornecer título, identificar o tema ou o assunto principal do texto, reproduzir, recontar, elaborar um resumo e responder perguntas sobre o texto. 
O método de fornecer título a um texto apresentado permite avaliar a capacidade de apreender o tema central ou o evento/aspecto principal do texto, o qual deve estar refletido no título fornecido. Utilizando esse método, Spinillo (2008) identificou três tipos de título que eram fornecidos pelas crianças: (a) inadequados; (b) apropriados, porém muito gerais; e (c) apropriados específicos. Observou-se que fornecer um título é atividade realizada com sucesso mesmo por crianças com dificuldades de compreensão de textos. Esse método é uma medida genérica que permite apenas identificar se a criança consegue ou não apreender o significado global de um texto. Outra limitação é que o título pode conter apenas o nome de algum personagem (no caso de histórias), pouco esclarecendo sobre a compreensão do leitor.

$\mathrm{O}$ método de identificar o tema ou o assunto principal tratado no texto avalia a compreensão global relativa à capacidade de identificar o que é relevante em um texto. Spinillo (2008) utilizou esse recurso ao investigar crianças com dificuldades de compreensão de textos, observando que o tema da história era facilmente identificado pelos participantes. Segundo nossa análise, esse recurso metodológico é pouco informativo, sendo aplicáveis aqui os comentários tecidos a respeito da tarefa de fornecer título. Nota-se, ainda, que muitas vezes as crianças acreditam que o assunto principal ou mais importante refere-se ao episódio ou ao personagem de que mais gostaram.

O método de reprodução requer que o indivíduo reproduza oralmente ou por escrito o texto que lhe foi apresentado. Em alguns estudos, a reprodução é feita com o apoio de uma sequência de gravuras ou de filmes (e.g., Guttman \& Frederiksen, 1985); em outros, nenhum apoio visual é fornecido (Brandão \& Spinillo, 1998; Salles \& Parente, 2004; Salles, Parente, Alexandre, Xavier, \& Fernandes, 2001). A análise consiste em dividir o texto original em proposições (ou em blocos de informação) e verificar se tais proposições estão presentes de forma literal ou parafraseada no texto reproduzido. Para que esse método seja, de fato, uma medida de compreensão, é fundamental que a reprodução não seja uma avaliação da capacidade de relembrar informações literais, mas sim uma forma de verificar se as informações implícitas no texto original foram explicitadas no texto reproduzido. Brandão e Spinillo (1998) classificaram as reproduções orais de crianças de 4 e 6 anos em categorias que expressam diferentes níveis de compreensão que variam quanto à sua aproximação com o texto original (não omitir informações principais e nem tampouco inserir informações ausentes no texto original) e quanto à presença das inferências. Salles e Parente (2004) também usaram esses critérios, porém definiram uma lista de proposições que variavam em função da relevância que tinham no texto. Essa análise gerou um quadro bastante preciso acerca da capacidade de compreensão dos leitores. O método de reprodução permite acessar a compreensão global do texto, a integração de informações nele veiculadas e a capacidade de selecionar informações relevantes. Uma das vantagens desse método é poder ser aplicado na modalidade oral em estudos com pessoas que não dominam a escrita.

No método de elaborar resumo, o participante é solicitado a escrever um resumo do texto que lhe foi apresentado. $\mathrm{O}$ resumo é considerado por muitos um indicador da compreensão (Curran, Kintsch, \& Hedberg, 1996; Cumming, Rebuffot, \& Ledwell, 1989; Rumelhart, 1977). Kintsch e Kozminsky (1977) compararam a escrita de resumos por universitários em duas condições: após lerem e após ouvirem histórias. As duas condições pouco diferiram, sendo isso interpretado como indicador de que existe um ponto comum do processo de compreensão que está presente tanto no ato de ler como no ato de ouvir um texto. Esse dado interessa a pesquisadores que investigam a compreensão de textos em leitores iniciantes (crianças e de jovens e adultos pouco escolarizados) ou que investigam a compreensão de textos desvinculada da habilidade de decodificação.

Os métodos de reprodução e de resumo se assemelham quanto ao fato de requererem: (a) habilidade de produção textual, no caso, conhecimentos sobre a estrutura do texto; (b) considerar a sequência de episódios e ideias do texto; (c) esforço de memória episódica, sobretudo quanto a textos narrativos; e (d) capacidade de selecionar as informações relevantes do texto. Portanto, tais atividades demandam habilidades que não estão associadas unicamente à compreensão, mas à produção textual.

Um método pouco utilizado na literatura é o de solicitar que o indivíduo elabore uma pergunta sobre um texto apresentado, partindo-se da ideia de que quem compreende um texto é capaz de nele identificar as informações cruciais que, nesse caso, poderiam se tornar respostas a determinadas perguntas. Após a leitura de uma história, Spinillo (2003) solicitou que crianças elaborassem uma pergunta sobre uma história lida, como se fosse um professor que desejasse saber se seus alunos haviam, de fato, entendido aquela história. Dois tipos de perguntas foram obtidos: perguntas que não se referiam ao conteúdo do texto ("Quantas linhas tem nessa história?"; "Quem escreveu essa história?") e perguntas de conteúdo voltadas para informações que estavam implícitas ou explicitas no texto, podendo ser relevantes ou irrelevantes. Como se nota, o participante pode ter em mente outras razões para ter elaborado uma dada pergunta, como, por exemplo, repetir perguntas típicas do contexto escolar, as quais seriam pouco informativas quanto à capacidade de compreender da criança.

O método de pergunta-resposta é amplamente utilizado nas pesquisas na área, consistindo em responder perguntas sobre um texto que é apresentado oralmente ou por escrito. Em termos de forma de apresentação, as perguntas podem ser do tipo verdadeiro/falso, abertas ou de múltipla escolha. Em termos do que tratam, as perguntas podem ser de conteúdo ou de explicação.

As perguntas do tipo verdadeiro/falso consistem em apresentar uma proposição relativa ao conteúdo tratado no texto e perguntar ao participante se a afimação é verdadeira ou falsa (Cain \& Oakhill, 2006). As desvantagens desse método, como comenta Cadime (2011), são que a probabilidade de acerto devido ao acaso é alta e que esse formato não permite avaliar o estabelecimento de inferências, pois, na maioria das vezes, as proposições referem-se a informações literais. Entretanto, essa limitação pode ser superada se informações inferenciais forem consideradas nas proposições apresentadas (ver Yuill e Oakhill,1991).

As perguntas de múltipla escolha consistem em perguntas de conteúdo cuja resposta deve ser escolhida dentre três ou 
mais alternativas (ver Snow, 2003). As possíveis alternativas precisam ser definidas, de maneira que representem a forma do leitor lidar com as informações literais do texto e com as inferências possíveis de serem estabelecidas, sendo que a exclusão de determinadas alternativas não seja evidente.

Tanto o método verdadeiro/falso como o de múltipla escolha exigem pouco esforço da memória, pouca habilidade verbal (podem ser aplicados a pessoas com limitações orais e escritas) e são de fácil aplicação e correção. Uma crítica que se aplica a ambos os métodos é que seus formatos envolvem mais o reconhecimento de sentenças do que os significados que efetivamente o leitor constrói a partir do texto. Nessa mesma linha de reflexão, considera-se que as proposições apresentadas são definidas a partir da leitura do próprio pesquisador. Assim, o papel do leitor é pouco ativo, e, dependendo de como forem elaboradas as perguntas e as alternativas, os dados derivados desses métodos pouco esclarecem acerca do estabelecimento de inferências.

No método de perguntas abertas, a resposta é fornecida pelo participante, requerendo que o pesquisador esteja preparado para lidar com respostas que podem estar corretas ou incorretas. Uma vantagem é a possibilidade de identificar problemas de compreensão a partir da análise da natureza das respostas incorretas fornecidas, bem como de explorar os diferentes significados que podem ser atribuídos a um mesmo texto a partir da análise das respostas corretas apresentadas. Em termos do que tratam, as perguntas abertas podem ser de conteúdo ou de explicação. Perguntas abertas de conteúdo são muito utilizadas, podendo versar sobre informações literais ou inferenciais (Brandão \& Spinillo, 1998, 2001; Cain \& Oakhill, 2004; Ferreira \& Dias, 2004; Oakhill \& Yuill, 1996; Yuill \& Oakhill, 1991).

As informações inferenciais podem estar associadas a informações que já foram veiculadas ou a informações que ainda serão apresentadas no texto. Este tipo é denominado de inferências de previsão (ver Solé, 1998), que apenas podem ser investigadas quando o texto é apresentado de forma interrompida (metodologia on-line), como discutido adiante. Spinillo e Mahon $(2007,2015)$ classificaram as respostas a perguntas de previsão em improváveis, que extrapolam ou são incoerentes com o sentido do texto, e em prováveis, que são plausíveis e mantêm coerência com o que foi até então veiculado no texto.

De modo geral, as respostas dadas a perguntas de conteúdo são analisadas como corretas ou incorretas. Contudo, Brandão e Spinillo (1998, 2001) classificaram as respostas em incongruentes, gerais e específicas. Os dois últimos tipos referem-se a respostas corretas, porém com níveis distintos de precisão. Esse tipo de análise permite identificar nuances que a dicotomia correto-incorreto não captura, tendo o pesquisador, de antemão, que decidir quais os possíveis significados autorizados pelo texto que podem ser considerados apropriados. Uma análise dessa natureza pressupõe que o texto possui múltiplos sentidos decorrentes da integração entre o conteúdo do texto e o conhecimento de mundo do leitor (Koch \& Elias, 2007; Marcuschi, 2008; Spinillo, 2013).

As perguntas de explicação, por sua vez, versam sobre as razões que levaram o indivíduo a fornecer uma determinada resposta, estando sempre apresentadas após uma pergunta ter sido respondida (ver Brandão \& Oakhill, 2005). Elas se voltam para as bases geradoras das respostas fornecidas e ainda podem servir como estratégias de leitura que favoreçam a compreensão do leitor, sobretudo quando associadas a uma metodologia on-line de investigação, como discutido adiante.

\section{Os Métodos em Função do Momento em que a Compreensão é Avaliada}

Considerando o momento em que a compreensão é avaliada, identifica-se o método off-line e o método on-line de investigação. O método off-line é o mais usual e investiga a compreensão após a leitura de todo o texto, enquanto o método on-line examina a compreensão durante a leitura. No método on-line, faz-se uma leitura interrompida do texto, o qual é segmentado em partes que são apresentadas uma a uma. Após a leitura de cada passagem, são feitas perguntas de conteúdo sobre o que foi lido até então ou perguntas sobre o que o leitor acredita que virá a seguir (pergunta de previsão). Apenas através do método on-line é possível investigar as inferências de previsão.

Spinillo e Mahon (2007) investigaram a compreensão de textos em relação a diferentes tipos de inferências. Adotando a metodologia on-line, as autoras tornaram possível investigar as inferências de previsão, as quais envolvem muitas informações extratextuais e requerem a formulação de hipóteses sobre a continuidade da narrativa. Os dados mostraram que as crianças apresentam dificuldade em prever eventos que estão por acontecer.

A metodologia on-line foi também adotada em estudos subsequentes em que se investigava o papel desempenhado por diferentes situações de leitura sobre a compreensão. Em um desses estudos, Spinillo e Hodges (2012) analisaram os erros apresentados por crianças com dificuldades de compreensão de textos em duas situações: leitura com interrupção e sem interrupção. Após a leitura de todo o texto, os participantes respondiam perguntas sobre informações inferenciais. Apesar dos tipos de erros serem os mesmos em ambas as situações, a leitura interrompida propiciou tentativas de estabelecer inferências mais eficientes do que a leitura sem interrupções. Em outra investigação, Spinillo e Hodges (2013) verificaram que a metodologia on-line pode ser considerada uma estratégia de leitura que favorece a compreensão, especificamente quanto ao estabelecimento de inferências.

Para Keenan et al. (1990), uma limitação do método online é a impossibilidade de saber se as inferências também seriam estabelecidas na ausência das perguntas. Segundo nossa análise, esse mesmo comentário se aplica às perguntas off-line, pois é possível que o leitor só estabeleça a inferência quando a pergunta lhe foi dirigida, independentemente do momento em que a pergunta lhe é feita, ou seja, o instrumento gera as inferências em vez de apenas detectá-las. Essa discussão ilustra a afirmação de que o dado pode ser produto do método, o que não transforma o dado em algo de menor relevância empírica ou teórica. De fato, até o momento, não se dispõe de recursos metodológicos que possam detectar se a inferência foi estabelecida antes ou depois da pergunta ter 
sido feita, ou se foi estabelecida durante ou após a leitura de todo o texto.

Os métodos on-line permitem examinar processos e estratégias adotadas pelos leitores, enquanto os off-line são mais voltados à avaliação do produto final. Ao avaliar o produto através de métodos off-line, o pesquisador obtém informações sobre níveis de compreensão, o que lhe permite comparar grupos, correlacionar a compreensão a outras habilidades e examinar o efeito de fatores sobre a compreensão. Por outro lado, ao avaliar o processo através de métodos on-line é possível obter informações acerca do que impede ou dificulta a compreensão e da dinâmica própria do ato de compreender um texto (alterações das representações mentais, das hipóteses mantidas ou descartadas durante a leitura etc.).

\section{Discussões e Considerações Finais}

O presente artigo procurou analisar as características, os limites e as possibilidades de diferentes recursos metodológicos adotados na pesquisa sobre compreensão de textos em crianças, classificando-os em função da unidade linguística investigada e do momento da leitura do texto. Esses dois critérios não são excludentes: eles se referem a características que podem se combinar na constituição de cada um dos métodos de investigação considerados.

Classificar os métodos a partir das unidades linguísticas permite identificar se a investigação examina aspectos globais ou locais da compreensão. Por exemplo, reproduzir um texto e elaborar um resumo são técnicas que avaliam uma compreensão global; por outro lado, o reconhecimento de palavras e a técnica de cloze se voltam para aspectos mais pontuais do texto. Essa classificação permite também identificar se a investigação focaliza mais os aspectos relativos a processos básicos (como a decodificação e o vocabulário) ou se a pesquisa volta-se mais para processos de alto nível (como as inferências). As inferências podem ser examinadas de forma mais local ou mais global, a depender do recurso metodológico empregado. A técnica de cloze, por exemplo, permite investigar as inferências de forma local, enquanto recursos metodológicos no âmbito do texto permitem investigar de modo mais efetivo as relações entre informações intra e extratextuais na compreensão.

Um aspecto inovador acerca das reflexões aqui conduzidas refere-se à análise da natureza das perguntas. A tendência dos estudiosos é classificar as perguntas em função da informação veiculada no texto: inferenciais e literais. No entanto, as perguntas podem versar sobre os processos de raciocínio que o leitor aciona ao interagir com o texto, como é o caso das perguntas de explicação que tornam possível examinar as bases geradoras das inferências: se preferencialmente oriundas de informações intratextuais ou de informações extratextuais. Outro ponto a considerar é que, quando a sperguntas de explicação estão associadas à metodologia on-line (ver Spinillo \& Hodges, 2013), assumem o status de estratégias que podem auxiliar a compreender textos. Spinillo (2010) aponta a relação entre compreensão de textos e tomada de consciência relativa ao estabelecimento de inferências, sendo essa tomada de consciência ativada por perguntas de explicação, concluindo-se que tais perguntas tanto podem servir para avaliar a compreensão como para desenvolvê-la.

Ao classificar os métodos em on-line e off-line, o foco recai sobre a análise do processo e do produto. Essa reflexão tem raízes nas discussões de Graesser e Zwaan (1995) sobre a origem das inferências: se são estabelecidas durante ou após a leitura. Pensar os métodos de pesquisa sob essa ótica torna possível investigar novos aspectos da compreensão de textos, como é o caso das inferências de previsão, que só podem ser examinadas a partir de uma metodologia on-line.

Diante dessas considerações, conclui-se que um único método não é suficiente para abarcar a multiplicidade de aspectos imbricados na ação de compreender um texto. Nesse sentido, a combinação de vários métodos em uma mesma investigação pode fornecer informações que se complementam.

Importante ressaltar que a escolha do recurso metodológico depende da perspectiva teórica do pesquisador. Observa-se que estudiosos afiliados a abordagens mais discursivas tendem a investigar a compreensão exclusivamente a partir da unidade linguística texto, enquanto pesquisadores afiliados a abordagens cognitivas examinam tanto o texto como um todo como unidades linguísticas, como a palavra e a sentença.

Portanto, os métodos de investigação são instâncias associadas a pressupostos teóricos que permitem tanto revelar aspectos distintos de um dado fenômeno como constituir tais aspectos, sendo inevitável a relação entre pesquisa e dado. O método mais indicado seria aquele coerente com a perspectiva teórica adotada, que atende as demandas e a proposta do pesquisador. Este, precisa ter clareza das razões por que adota tais recursos metodológicos, estando ciente dos limites e possibilidades que suas escolhas metodológicas representam.

\section{Referências}

Balota, D. A. (1990). The role of meaning in word recognition. In D. A. Balota, G. B. Flores D'Arcais, \& K. Rayner (Eds.), Comprehension processes in reading (pp. 9-32). Hillsdale: Lawrence Erlbaum.

Brandão, A. C. P., \& Oakhill, J. (2005). "How do you know this answer?" Children's use of text data and general knowledge in story comprehension. Reading and Writing, 18(7-9), 687-713.

Brandão, A. C. P., \& Spinillo, A. G. (1998). Aspectos gerais e específicos na compreensão de textos. Psicologia: Reflexão e Critica, 11(2), 253-272.

Brandão, A. C. P., \& Spinillo, A. G. (2001). Produção e compreensão de textos em uma perspectiva de desenvolvimento. Estudos de Psicologia, 6(1), 51-62.

Cadime, I. M. D. (2011). Avaliar leitura no $1^{\circ}$ ciclo do ensino básico: Construção e validação do TCL - teste de Compreensão Leitora (Unpublished doctor dissertation). Escola de Psicologia, Universidade do Minho, Braga.

Cain, K., \& Oakhill, J. (2004). The develop of comprehension skills. In P. E. Bryant \& T. Nunes (Eds.), Handbook of children's literacy (pp. 155-338). Dordrecht: Klover Academic. 
Cain, K., \& Oakhill, J. (2006). Assessment matters: Issues in the measurement of reading comprehension. British Journal of Educational Psychology, 76, 697-708.

Colomer, T., \& Camps, A. (2002). Ensinar a ler, ensinar a compreender. Porto Alegre: Artmed.

Cumming, A., Rebuffot, J., \& Ledwell, M. (1989). Reading and summarizing challenging texts in first and second languages. Reading and Writing, 1(3), 201-219.

Curran, C. E., Kintsch, E., \& Hedberg, N. (1996), Learning-disabled adolescents' comprehension of naturalistic narratives. Journal of Educational Psychology, 88(3), 494-507.

Drum, P. A., Calfee, R. C., \& Cook, L. K. (1981). The effects of surface structure variables on performance in reading comprehension tests. Reading Research Quarterly, 16, 486514.

Ferreira, S. P. A., \& Dias, M. G. B. B. (2004). A leitura, a produção de sentidos e o processo inferencial. Psicologia em Estudo, 9, 439-448.

Forster, K. I. (1981). Priming and the effects of sentence and lexical contexts on naming time: evidence for autonomous lexical processing. Quarterly Journal of Experimental Psychology, 33, 465-495.

Gough, P. B., Hoover, W. A., \& Peterson, C. L. (1996). Some observations on a simple view of reading. In C. Cornoldi \& J. Oakhill (Eds.), Reading Comprehension difficulties: Processes and intervention (pp. 1-13). Mahwah: Lawrence Erlbaum Associates.

Graesser, A., \& Zwaan, R. (1995). Inference generation and the construction of situation models. In C. Weaver, S. Mannes, \& C. Fletcher (Eds.), Discourse comprehension: Essays in honor of Walter Kintsch (pp. 117-139). Hillsdale: Lawrence Erlbaum Associates.

Guttman, M., \& Frederiksen, C. H. (1985). Preschool children's narratives: Linking story comprehension, production and play discourse. In L. Galda \& A. D. Pellegrini (Eds.), Play, language and stories: the development of children's literate behavior (pp. 69-92). Norwood: Ablex.

Haviland, S. E., \& Clark, H. H. (1974). What's new? Acquiring new information as a process in comprehension. Journal of Verbal Learning and Verbal Behavior, 13, 512-521.

Keenan, J. M., Potts, G. R., Golding, J. M., \& Jennings, T. M. (1990). Which elaborative inferences are drawn during reading? A question of methodologies. In D. A. Balota \& G. B. Flores d'Arcais (Eds.), Comprehension processes in reading (pp. 377-402). Hillldale: Lawrence Erlbaum Associates.

Kintsch, W. (1998). Comprehension: A paradigm for cognition. Cambridge: Cambridge University Press.

Kintsch, W., \& Kozminsky, E. (1977). Summarizing stories after reading and listening. Journal of Educational Psychology, 69(5), 491-499.

Kleiman, A. (2002). Texto \& leitor: Aspectos cognitivos da leitura. Campinas: Pontes.

Koch, I. V., \& Elias, V. M. (2007). Ler e compreender: Os sentidos do texto. São Paulo: Contexto.

Marcuschi, L. A. (2008). Processos de produção textual. In L. A. Marcuschi (Ed.), Produção textual, análise de gêneros e compreensão (pp. 50-61). São Paulo: Parábola.
Oakhill, J., \& Cain, K. (2007). Issues of causality in children's reading comprehension. In D. S. McNamara (Ed.), Reading comprehension strategies: Theories, interventions, and technologies (pp. 47-71). New York: Lawrence Erlbaum.

Oakhill, J., \& Yuill, N. (1996). Higher order factors in comprehension disability: Processes and remediation. In C.Cornoldi, \& J. Oakhill (Eds.), Reading comprehension difficulties: processes and intervention (pp. 69-92). Mahwah: Lawrence Erlbaum.

Oliveira, K. L., Boruchovitch, E., \& Santos, A. A. A. (2009). A técnica de cloze na avaliação da compreensão em leitura. In A. A. A. Santos, E. Boruchovitch, \& K. L. Oliveira (Eds.), Cloze: um instrumento de diagnóstico e intervenção (pp. 47-78). São Paulo: Casa do Psicólogo.

Oliveira, K. L., Boruchovitch, E., \& Santos, A. A. A. (2007). Compreensão de leitura em alunos de sétima e oitava séries do ensino fundamental. Psicologia Escolar e Educacional, 11, 41-49.

Rumelhart, D. E. (1977). Understanding and summarizing brief stories. In D. LaBerge \& S. J. Samuels (Eds.), Basic process in reading: Perception and comprehension (pp. 265-303). Hillsdale, NJ: Lawrence Erlbaum.

Salles, J. F., \& Parente, M. A. M. P. (2002). Processos cognitivos na leitura de palavras em crianças: Relações com compreensão e tempo de leitura. Psicologia: Reflexão e Crítica, 15(2), 321-331.

Salles, J. F., \& Parente, M. A. M. P. (2004). Compreensão textual em alunos de segunda e terceira séries: Uma abordagem cognitiva. Estudos de Psicologia (Natal), 9(1), 71-80.

Salles, J. F., Parente, M. A. M. P., Alexandre, B., Xavier, C., \& Fernandes, J. (2001). Recontar de histórias por crianças: Instrumentos de avaliação da compreensão de leitura. Letras de Hoje, 36(3), 529-535.

Santos, A. A. A., Boruchovitch, E., \& Oliveira, K. L. (2009). Cloze: Um instrumento de diagnóstico e intervenção. São Paulo: Casa do Psicólogo.

Santos, A. A., Primi, R., Taxa, F. de O. S., \& Vendramini, C. M. M. (2002). O teste de cloze na avaliação da compreensão em leitura. Psicologia: Reflexão e Crítica, 15(3), 549-560.

Snow, C. E. (2003). Assessment of reading comprehension: Researchers and practitioners helping themselves and each other. In A. P. Sweet \& C. E. Snow (Eds.), Rethinking reading comprehension (pp.192-206). New York: The Guilford Press.

Solé, I. (1998). Estratégias de leitura. Porto Alegre: Artmed.

Spinillo, A. G. (2008). O leitor e o texto: Desenvolvendo a compreensão de textos na sala de aula. Revista Interamericana de Psicologia, 42(1), 29-49.

Spinillo, A. G. (2003). Compreensão de textos em crianças: Um estudo de intervenção (Relatório de pesquisa CNPq). Recife: Universidade Federal de Pernambuco.

Spinillo, A. G. (2010, Julho). Compreensão de textos e metacognição: O papel da tomada de consciência no estabelecimento de inferências. In $8^{\circ}$ Encontro Nacional $\mid 6^{\circ}$ Internacional de Investigação em Leitura, Literatura Infantil e Ilustração (pp. 42-57). Braga: Universidade do Minho.

Spinillo, A. G. (2013). A dimensão social, linguística e cognitiva da compreensão de textos: Considerações teóricas e aplicadas. In M. Mota \& A. G. Spinillo (Eds.), Compreensão de texto: Contribuições da psicologia cognitiva (pp. 171-198). São Paulo: Casa do Psicólogo. 
Spinillo, A. G., \& Hodges, L. V. S. D. (2012). Análise de erros e compreensão de textos: Comparações entre diferentes situações de leitura. Psicologia: Teoria e Pesquisa, 28(4), 381-388.

Spinillo, A. G., \& Hodges, L. V. S. D. (2013). Situações de leitura e o estabelecimento de inferências por crianças com dificuldades de compreensão de textos. Em A. Roazzi; F.R. dos R. Justi, \& J. F. de Salles. (Eds.). A aprendizagem da leitura e da escrita: Contribuições de pesquisas. (pp. 131-164). São Paulo: Editora Vetor.

Spinillo, A. G., \& Mahon, E. (2007). Compreensão de texto em crianças: Comparações entre diferentes classes de inferência a partir de uma metodologia on-line. Psicologia: Reflexão $e$ Crítica, 20(3), 463-471.

Spinillo, A.G., \& Mahon, E. da R. (2015). "O que você acha que vai acontecer agora?” Um estudo sobre as inferências de previsão na compreensão de textos. Em A. C. Naschold; A. Pereira Jr; R. Guaresi, \& V. W. Pereira (Eds.), Aprendizado da leitura e da escrita: A ciência em interface. (pp. 163-192). Natal: Editora da Universidade Federal do Rio Grande do Norte
Stanovich, K. E., West, R. F., Cunningham, A. E., Cipielewski, J., $\&$ Siddiqui, S. (1996). The role of inadequate print exposure as a determinate of reading comprehension problems. In C. Cornoldi \& J. Oakhill (Eds.), Reading comprehension difficulties: Processes and intervention (pp.15-32). Mahwah: Lawrence Erlbaum.

Tunmer, W. E., Nesdale, A. R., \& Wright, A. D. (1987). Syntactic awareness and reading acquisition. British Journal of Developmental Psychology, 5, 25-34.

Yuill, N., \& Oakhill, J. (1991). Children's problems in text comprehension: An experimental investigation. Cambridge: Cambridge University Press.

Recebido em 09.07.2013

Primeira decisão editorial em 11.06.2015

Aceito em 11.06.2015 